\title{
The thyroid gland in postmenopausal women: physiology and diseases
}

\author{
Małgorzata Gietka-Czernel \\ Department of Endocrinology, Centre of Postgraduate Medical Education, Bielański Hospital, Warsaw, Poland
}

\begin{abstract}
The incidence of most thyroid diseases: hypothyroidism, nodular goitre, and cancer is highest among postmenopausal and elderly women. The diagnosis of thyroid dysfunction in this group of patients is difficult because the symptoms can be nonspecific or common with menopausal and ageing complaints. In the interpretation of thyroid function tests the physiological changes in secretion and metabolism of thyrotropin (TSH) and thyroid hormones must be considered, as well as the influence of comorbidities. Unrecognised thyroid dysfunction leads to increased: cardiovascular risk, bone fractures, cognitive impairment, depression, and mortality. Therapy of thyroid dysfunction is different in postmenopausal and elderly women than in young people; hypothyroidism should be treated with caution, because high doses of L-thyroxine can lead to cardiac arrhythmias and increased bone turnover, and hyperthyroidism should be preferentially treated with radioiodine. Thyroid status beneficially influencing longevity relates to low thyroid function.

Thyroid nodules and cancer often affect women over 50 years old; the diagnostic and therapeutic approach is the same as in the general population, but the surgical risk and cancer prognosis is worse than in young patients.
\end{abstract}

Key words: menopause, thyroid physiological changes, thyroid diseases.

\section{Introduction}

Thyroid diseases predominantly affect women; their incidence is 5-20 times higher in women than in men. Additionally, the prevalence of most thyroid diseases increases with age. Consequently, thyroid gland autoimmunity, hypothyroidism, nodular goitre, and cancer occur most often in postmenopausal and elderly women. The diagnosis of thyroid disease is difficult in this group of patients because the symptoms like anxiety, heart palpitations, sweating, gaining weight and insomnia are common for the both thyroid and ovarian dysfunction. The additional problems arise from the interpretation of the results of thyroid function tests: according to many observations the serum TSH, thyroxine (T4), and tri-iodothyronine (T3) concentrations depend on age, comorbidities, and medical treatment - these together sometimes make the diagnosis of thyroid dysfunction complicated in older population. Although thyroid status has a well-known impact on cardiovascular risk, cognitive function, disability, and longevity there is no consensus on universal screening for thyroid dysfunction of postmenopausal and elderly women among medical associations worldwide [1].

\section{Physiological changes in ageing thyroid}

According to morphological studies, thyroid epithelium undergoes degenerative processes that lead to its flattening, the size of thyroid follicles diminishes, while fibrous connective tissue and lymphoid tissue proliferates. In consequence, the size of the thyroid gland may reduce over time. The ability of the thyroid gland to uptake iodine diminishes, and in people over 80 years old it becomes $40 \%$ lower than in people 30 years old. The daily production of the main thyroid hormone $\mathrm{T} 4$ decreases per $20 \mu \mathrm{g}$, but at the same time its metabolism slows down due to the reduced activity of 5' deiodinase-I. As a result, the half-life of T4 elongates from 8 to 9.3 days, and the serum concentration of T4 does not change over time. The daily production of $\mathrm{T} 3 \mathrm{dimin}$ ishes per $20 \mu \mathrm{g}$ in men and $10 \mu \mathrm{g}$ in women, and the serum concentration of T3 in elderly people decreases significantly. Studies evaluating a hypothalamic-pituitary-thyroid axis demonstrate different patterns of pituitary function: its preserved or diminished ability to secrete TSH after various stimuli [2].

According to several studies, serum TSH concentration is probably age-dependant. The observations conducted in iodine-sufficient areas have shown that serum TSH concentrations rise with age in both men and women. In the NHANES III survey (National Health and Nutrition and Examination Survey III) evaluation of the American reference population negative for thyroid diseases, anti-thyroid antibodies and medications influencing thyroid function demonstrated an increase in mean, median, and 97.5 
percentile for TSH concentration with age. The analysis has shown that the 97.5 percentile is $3.6 \mathrm{mIU} / \mathrm{l}$ in people 20-29 years of age, and 4.03 and $5.9 \mathrm{mIU} / \mathrm{l}$ in those who are 50-59 and 70-79 years old, respectively [3].

Similar results have been obtained in the Busselton study from Western Australia: the upper limit of TSH is $3.5 \mathrm{mIU} / \mathrm{l}$ in people < 30 years old, and 4.09 and $5.28 \mathrm{mIU} / \mathrm{l}$ in those who are 50-60 and > 70 years old, respectively [4]. These data may suggest a diminished pituitary sensitivity to T4 in the ageing population. Contrasting observations were made in the areas of previously deficient and borderline sufficient iodine intake, indicating that TSH decreases with age. According to a European study made in a population free of thyroid diseases, anti-thyroid antibodies, and abnormalities on thyroid ultrasound, the mean serum TSH concentration is $1.47 \mathrm{mIU} / \mathrm{l}$ in people $18-24$ years of age and $1.02 \mathrm{mIU} / \mathrm{l}$ in participants aged over 80 years [5]. Another observation performed on the Pomeranian population demonstrated a reference range for TSH 0.25-2.1 $\mathrm{mIU} / \mathrm{l}$ [6]. The probable explanation of these findings is the development of thyroid autonomy after longstanding iodine deficiency. We can hypothesise that in the Polish population born before implementation of the iodine prophylaxis programme in 1997 the TSH concentration will decrease with age, but in the future, in people born after 1997, TSH will increase with age, similarly to the American population. This short review demonstrates that the interpretation of thyroid function tests (TFTs) may be complicated in the ageing population, and especially the diagnose of subclinical hypothyroidism should be made with caution. An additional problem may be posed by the various medications used by older people, which can influence TFTs, for example metformin and glycocorticosteroids, which decrease serum TSH concentration, or amiodarone, which has a multidirectional effect on serum TSH and thyroid hormone levels.

\section{Hypothyroidism}

Hypothyroidism, especially its subclinical form, is one of the most commonly occurring endocrinopathies. It affects women 7-10 times more frequently than men, and its rate increases with age. According to the Whickham survey, the incidence of elevated TSH is about $7.6 \%$ in the general female population but rises to $17 \%$ in women over 70 years of age. The risk for developing hypothyroidism is $1.4 / 1000$ /year in women $18-24$ years of age, 6.7/1000/year in women 65-74 years of age, and $14 / 1000 /$ year in women $75-80$ years of age $[7,8]$. In the Colorado survey the incidence of subclinical hypothyroidism is even higher: about 4\% in women aged 25 years, and rises to 14 and $20 \%$ in women aged 55 and 75 years, respectively [9]. The most common cause of hypothyroidism is chronic autoimmune thyroiditis (syn. Hashimoto thyroiditis), previous treatment with radioiodine and sur- gery for benign or malignant thyroid diseases, external beam irradiation of malignant tumours of the head and neck, and several drugs, such as lithium, amiodarone, interferon, and tyrosine kinase inhibitors.

The typical symptoms of overt hypothyroidism (serum TSH elevated; freeT4 decreased) are dry skin, poor memory, slower thinking, somnolence, tiredness, muscle cramps, feeling cold, hoarse voice, general oedema, and constipation. All of them diminish the quality of life and working capacity. Hypothyroidism causing hypertension, cardiac insufficiency, adverse lipid profile, insulin resistance, and endothelial dysfunction poses an increased risk for atherosclerosis, cardiovascular disease, diabetes mellitus t.2, cognitive impairment, depression, and mortality.

The symptoms of subclinical hypothyroidism (serum TSH elevated; fT4 within the normal range) may be very scarce (such as greater tiredness, drier skin, and weight gain) or even absent. In subclinical hypothyroidism, the risk of cardiovascular disease, cardiac insufficiency, and cardiovascular mortality seems to be associated with $\mathrm{TSH} \geq 10 \mathrm{mIU} / \mathrm{l}$ and age $<65$ years. Several observations have documented not only the lack of cardiovascular risk, impairment of cognitive function, and mortality in population over 65-70 years of age, but also the protective role of subclinical hypothyroidism in elderly and longer survival [10-13].

The progression of subclinical to overt hypothyroidism is about $2.6 \%$ per year and can double in cases with a-TPO positivity or TSH > $10 \mathrm{mIU} /$. Also, spontaneous remission of subclinical hypothyroidism was observed, affecting as many as $37-46 \%$ of patients aged $\geq 65$ years during 2-4 years of follow-up [14].

Treatment of hypothyroidism in post-menopausal women depends on several conditions: the presence of symptoms, severity of hypothyroidism, coexisting cardiovascular risk, and patient's age. In overt hypothyroidism, treatment with L-thyroxine is always recommended. In patients with cardiovascular risk and the elderly the starting dose should be low: $25 \mu \mathrm{g}$ daily and increased slowly every 2-4 weeks by an additional 12.5-25 $\mu \mathrm{g}$ of L-thyroxine daily. Women taking hormone replacement therapy need the higher doses of L-thyroxine due to the greater capacity of the main thyroxine protein blood transporter - TBG (thyroxine-bounding globulin). The therapy of subclinical hypothyroidism is a matter of controversy. According to the European Thyroid Association guidelines, in patients with subclinical hypothyroidism and age $<65-70$ years, therapy with L-thyroxine should be instituted in the presence of symptoms or $\mathrm{TSH} \geq 10 \mathrm{mIU} / \mathrm{l}$. In the population over 70 years old and TSH $\geq 10 \mathrm{mIU} / \mathrm{l}$, therapy may be considered when symptoms of hypothyroidism or cardiovascular risk exist. In patients $>70$ years of age and $\mathrm{TSH}<10 \mathrm{mIU} / \mathrm{l}$, observation and TFTs every six months should be conducted [15]. 
In the opinion of other authors, L-thyroxine should be implemented even in slightly elevated serum TSH $5-<10 \mathrm{mIU} / \mathrm{l}$ if there is a cardiovascular risk (cardiac diastolic insufficiency, diastolic hypertension, diabetes mellitus, adverse lipid profile, nicotinism), goitre, antiperoxidase antibodies (a-TPO) positivity, and thyroid ultrasound scan consistent with chronic lymphocytic inflammation [16]. When L-thyroxine therapy is instituted, the target TSH depends on the patient's age and is recommended to be: $0.5-2.0 \mathrm{mIU} / \mathrm{l}$ in young people, $1.0-3.0 \mathrm{mIU} / \mathrm{l}$ in patients around 50 years old, and 2-5 mIU/l in patients 70-80 years old [17].

In a recent double-blind, randomised, placebo-controlled trial involving 737 adults who were at least 65 years of age (mean 74.4 years) and who had persisting subclinical hypothyroidism (TSH level 4.60 to $19.99 \mathrm{mIU} / \mathrm{l}$; free thyroxine level within the reference range), no apparent benefits in alleviation of symptoms were obtained during one-year observation [18]. In a retrospective study evaluating the effect of L-thyroxine therapy for subclinical hypothyroidism on cardiovascular risk and mortality, the only beneficial effect was observed in the population aged $40-70$ years and was even deleterious in those aged over 80 years [19].

One must be aware that overtreatment with L-thyroxine leading to suppressed TSH is associated with increased risk of atrial fibrillation, coronary heart disease events, and significant bone loss in postmenopausal women.

\section{Hyperthyroidism}

Hyperthyroidism occurs in $2 \%$ of the general population with 10-20-time preponderance of women to men. The incidence and aetiology of hyperthyroidism depends on iodine intake. In areas with adequate iodine supply the main cause of hyperthyroidism is Graves' disease. In the Whickham epidemiological survey hyperthyroidism was observed in $3.9 \%$ of adult women and $0.2 \%$ of men $[7,8]$. The incidence of disease was highest among women aged 18-24 years, at $2.2 / 1000 / y e a r$, and in the rest of the female population until 80 years of age it was $<1 / 1000 /$ year. $40 \%$ of cases of hyperthyroidism were noted before 45 years of age and 38\% among women aged 45-64 years. In areas of iodine deficiency, the main cause of hyperthyroidism is toxic nodular goitre, which affects predominantly the older population. In an Italian study undertaken in an iodine deficient area, toxic nodular goitre was noted in $6.4 \%$ of the general population and in $15 \%$ of people over 75 years of age [20]. In Poland recently the dominant cause of hyperthyroidism has been toxic nodular goitre; from 1997, after the iodine prophylaxis programme was instituted, the frequency of toxic nodular goitre has been gradually diminishing while Graves' disease will increase.
One must be aware of the fact that in the older population the higher incidence of drug-induced hyperthyroidism occurs in the following cases: post amiodarone, iodine-containing contrast media, excessive amounts of L-thyroxine.

The symptoms of hyperthyroidism can be mistaken for those of menopause: anxiety, increased sweating, heart palpitations, and insomnia are common for the two entities. In differential diagnosis, the important symptoms of hyperthyroidism are: weight loss, cardiac arrhythmias, especially atrial fibrillation and goitre, orbitopathy, and pretibial oedema due to Graves' disease. Overt hyperthyroidism (suppressed serum TSH; elevated T3 and/or T4) is associated with increased risk of ischaemic heart disease (IHD), atrial fibrillation, cardiac dysfunction, and total and IHD mortality. The disease is a well-known cause of osteoporotic fractures in post-menopausal women and dementia in the elderly. The consequences of subclinical hyperthyroidism (SH) (suppressed TSH; T3 and T4 within the normal range) to human health depends upon its severity and the patient's age. When the serum TSH concentration is less than $0.1 \mathrm{mIU} / \mathrm{I}$ (grade II SH) the consequences are similar to those of overt hyperthyroidism. In cases with TSH 0.1-0.3 $\mathrm{mIU} / \mathrm{I}$ (grade I SH) the increased risk of atrial fibrillation and dementia were confirmed [21]. A study of community-dwelling people over 60 years of age conducted in Great Britain showed that participants with $\mathrm{TSH}<0.5 \mathrm{mIU} / \mathrm{l}$ at baseline had a circulatory mortality $10 \%$ higher than euthyroid participants during five years of longitudinal observation [22].

Aside from the long-term health outcomes, $\mathrm{SH}$ can progress to overt hyperthyroidism. In grade II SH the risk is 2-5\% per year, and in grade I SH the rate of progression is very low. People with Graves' disease are more likely to progress than those with nodular goitre.

On the contrary, SH may subside spontaneously over time. The study by Parle et al. showed that TSH returned to normal in $76 \%$ of people aged $\geq 60$ years with low but detectable TSH during one year of follow-up [23].

Therapy of overt hyperthyroidism is always recommended whether the patient is symptomatic or not; the preferential form of treatment is radioiodine, although anti-thyroid drugs in Graves' disease and surgery in toxic nodular goitre may be considered. In grade II SH a similar approach is recommended, especially in postmenopausal women who are at risk of osteoporosis. In grade I $\mathrm{SH}$ patients over 65 years of age, symptomatic, with heart disease or cardiac risk factors and risk of osteoporosis should be treated as well. In all groups of patients $\beta$-blocking drugs can ameliorate cardiac symptoms [24].

\section{Thyroid status and longevity}

The Leiden 85+ study conducted for five years in 599 participants aged 85-89 years showed that higher TSH 
concentration and lower fT4 levels were associated with longer survival. The participants with low TSH levels had the highest mortality rate [13]. The American study in elderly Ashkenazi Jews with median age 98 years demonstrated that centenarians had significantly higher TSH levels than Ashkenazi controls aged 72 years. The authors identified a heritable phenotype connected with raised TSH and longevity: a single nucleotide polymorphism in the TSH receptor gene rs12050077 and rs10149689 [25]. Consequently there was lower expression of TSH receptors and lower sensitivity of thyroid to TSH. The final effect was lower metabolic rate and caloric restriction. The observations of van den Beld et al. in the group of elderly men showed that low fT4 level (within the normal range) is associated with longer survival, and low T3 concentration determines better physical performance [26]. In the animal model the dependence between low thyroid activity and longevity was confirmed.

\section{Benign nodular thyroid disease}

There is a close connection between the incidence of benign nodular thyroid disease (syn. nodular goitre), age, and gender. In autopsy examinations, the incidence of thyroid nodules is $50-90 \%$ in women and $50-60 \%$ in men. According to ultrasonography examinations, thyroid nodules are found in $10 \%$ of people around 20 years of age and in $50 \%$ of those aged $>60$ years. In an iodine deficient Italian population, the frequency of benign nodular thyroid disease was even higher: $30 \%$ in young people and $75 \%$ in those aged 55-66 years. The goitre size increases with the age of the population studied.

There may be an extremely slow progression from non-toxic to toxic nodular goitre, which can accelerate after ingestion of large amounts of iodine.

The symptoms of disease vary: from the incidentally discovered thyroid nodules during ultrasonographic neck exploration to the very large deforming goitres with tracheal obstruction. In cases of substernal goitre, superior caval vein syndrome may develop. The attack of atrial fibrillation may be the first syndrome of evolving thyroid autonomy.

The treatment should be adequate to the medical problem: observation with TFTs and thyroid ultrasound every 1-3 years in small goitres or surgical and radioiodine therapy in large obstructive goitres. Fine-needle aspiration biopsy is a very important diagnostic tool assessing the oncologic risk of the disease [27].

\section{Thyroid cancer}

The incidence of differentiated thyroid cancer increases in the population over 55 years old, and the incidence of anaplastic thyroid cancer increases in those aged over 65 years. In elderly people a higher frequency of thyroid lymphoma and sarcoma is also noted. The genetic background of papillary carcinoma differs according to the age: in $40-60 \%$ of older patients activating BRAF mutation is observed, while in $50 \%$ of children and $25 \%$ of adults $<45$ years old RET/PTC rearrangement is the major genetic event leading to cancerogenesis.

The symptoms and treatment of thyroid cancer are the same in all adult patients: fine-needle aspiration biopsy is the most important pre-operative diagnostic approach, and surgery is a cornerstone of therapy.

Older patients have greater risk of surgery because of comorbidities and poorer tolerance of suppressive doses of L-thyroxine. People > 45 years old with differentiated thyroid cancer have a poorer prognosis than younger patients.

\section{Conclusions}

1. Thyroid diseases like hypothyroidism, nodular goitre, and cancer are especially frequent in postmenopausal and elderly women.

2. The diagnosis of thyroid dysfunction in this group of patients may be difficult because of nonspecific symptoms and different interpretation of thyroid function tests.

3. Thyroid dysfunction increases cardiovascular risk, and cardiovascular and general mortality, and hyperthyroidism leads to increased risk of osteoporotic fractures in postmenopausal women.

4. Low thyroid function relates to longevity.

5. Thyroid cancer and its treatment relate to worse outcome in postmenopausal women than in the young population.

\section{Disclosure}

Author reports no conflict of interest.

\section{References}

1. Garber JR, Cobin RH, Gharib H, et al. Clinical practice guidelines for hypothyroidism in adults: consponsored by Americam Association of Clinical Endocrinologists and the American Thyroid Association. Thyroid 2012; 22: 1200-1235.

2. Mariotti S, Franceschi C, Cosarizza A, et al. The ageing thyroid. Endocr Rev 1995; 16: 686-715.

3. Surks MI, Hollowell JG. Age-specific distribution of serum thyrotropin and antithyroid antibodies in the US population: implications for the prevalence of subclinical hypothyroidism. J Clin Endocrinol Metab 2007; 92: 4575-4582.

4. Bremner AP, Feddema P, Leedman PJ, et al. Age-related changes in thyroid function: a longitudinal study of a community-based cohort. J Clin Endocrinol Metab 2012; 97: 1554-1562.

5. Hoogendoorn EH, Hermus AR, de Vegt F, et al. Thyroid function and prevalence of anti-thyroperoxidase antibodies in a population with borderline sufficient iodine intake: influences of age and sex. Clinical Chemistry 2006; 52: 104-111.

6. Völzke H, Alte D, Kohlmann T, et al. Reference intervals of serum thyroid function tests in a previously iodine-deficient area. Thyroid 2005; 15 : 279-285. 
7. Tunbridge WMG, Evered DC, Hall R. The spectrum of thyroid disease in a community: the Whickham survey. Clin Endocrinol 1977; 7: 481-493.

8. Vanderpump MPJ, Tunbridge WMG, French JM, et al. The incidence of thyroid disorders in the community: a twenty-year follow-up of the Whickham Survey. Clin Endocrinol 1995; 43: 55-68.

9. Canaris GJ, Manowitz NR, Mayor G, et al. The Colorado thyroid disease prevalence study. Arch Intern Med 2000; 160: 526-534.

10. Razvi S, Shakoor A, Vanderpump MPJ, et al. The influence of age on the relationship between subclinical hypothyroidism and ischemic heart disease: a metaanalysis. J Clin Endocrinol Metab 2008; 93: 2998-3007.

11. Ochs N, Auer R, Bauer DC, et al. Meta-analysis: subclinical thyroid dys function and the risk for coronary heart disease and mortality. Ann Intern Med 2008; 148: 832-845.

12. Rodondi N, den Elzen WP, Bauer DC, et al. Subclinical hypothyroidism and the risk of coronary heart disease and mortality. JAMA 2010; 304: 1365-1374.

13. Gussekloo J, van Exel E, de Craen AJ, et al. Thyroid status, disability and cognitive function, and survival in old age. JAMA 2004; 292: 2591-2599.

14. Somwaru LL, Rariy CM, Arnold AM, et al. The natural history of subclinical hypothyroidism in the elderly: the cardiovascular health study. J Clin Endocrinol Metab 2012; 97: 1962-1969.

15. Pearce S, Brabant G, Duntas LH, et al. 2013 ETA Guideline: Management of subclinical hypothyroidism. Eur Thyroid J 2013; 2: 215-228.

16. Biondi B, Cooper DS. The clinical significance of subclinical thyroid dysfunction. Endocr Rev 2008; 29: 76-131.

17. Burch HB, Burman KD, Cooper DS, et al. A 2013 survey of clinical practice patterns in the management of primary hypothyroidism. J Clin Endocrinol Metab 2014; 99: 2077-2085.

18. Stott DJ, Rodondi N, Kearney PM, et al. Thyroid hormone therapy for older adults with subclinical hypothyroidism. N Engl J Med 2017. DOI: 10.1056/NEJMoa1603825.

19. Razvi S, Weaver JU, Butler TJ, et al. Levothyroxine treatment of subclinical hypothyroidism, fatal and nonfatal cardiovascular events and mortality. Arch Intern Med 2012; 172: 811-817.

20. Aghini-Lombardi F, Antonangeli L, Martino L, et al. The spectrum of thyroid disorders in an iodine-deficient community: the Pescopagano survey. J Clin Endocrinol Metab 1999; 84: 561-566.

21. Mitchell AL, Pearce SHS. How should we treat patients with low serum thyrotropin concentrations? Clin Endocrinol 2010; 72: 292-296.

22. Parle J, Maisonneuve P, Sheppard M, et al. A single low thyrotropin (TSH) concentration predicts increased all-cause and cardiovascular mortality in older persons in the community. A 10 year cohort study. Lancet 2001; 358: 861-865.

23. Parle J, Franklyn JA, Cross K, et al. Prevalence and follow-up of abnormal thyrotropin concentrations in the elderly in the United Kingdom. Clin Endocrinol 1991; 34: 77-83.

24. Biondi B, Bartalena L, Cooper DS, et al. The 2015 European Thyroid Association guidelines on diagnosis and treatment of endogenous subclinical hyperthyroidism. Eur Thyroid J 2015; 4: 149-163.

25. Atzmon G, Barzilai N, Surks M, et al. Genetic Predisposition to elevated serum thyrotropin is associated with exceptional longevity. J Clin Endocrinol Metab 2009; 94: 4768-4775.

26. van den Beld AW, Visser TJ, Feelders RA, et al. Thyroid hormone concentrations, disease, physical function, and mortality in elderly men. J Clin Endocrinol Metab 2005; 90: 6403-6409.

27. Bryan R, Haugen BR, Alexander EK, et al. 2015 American Thyroid Association management guidelines for adult patients with thyroid nodules and differentiated thyroid cancer. Thyroid 2016; 26: 1-133. 